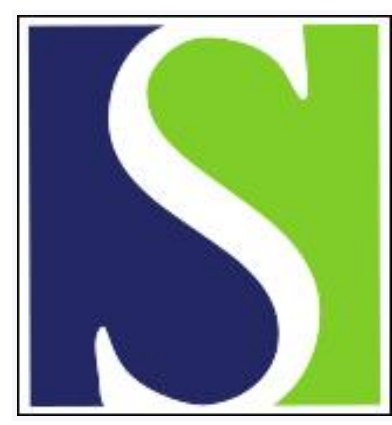

Scand J Work Environ Health 1991;17(4):281-285

https://doi.org/10.5271/sjweh.1701

Issue date: Aug 1991

Highlights of the 1990 Leesburg, Virginia, International Workshop on Retrospective Exposure Assessment for Occupational Epidemiology Studies.

by Stewart PA, Herrick RF, Blair A, Checkoway H, Droz P, Fine L, Fischer L, Harris R, Kauppinen T, Saracci R

Affiliation: Environmental Epidemiology Branch, National Cancer Institute, Rockville, Maryland.

This article in PubMed: www.ncbi.nlm.nih.gov/pubmed/1925441 


\section{Highlights of the 1990 Leesburg, Virginia, International Workshop on Retrospective Exposure Assessment for Occupational Epidemiology Studies}

by Patricia A Stewart MS, 1, 2 Robert F Herrick, ScD, 2,3 Aaron Blair, PhD, ${ }^{1}$ Harvey Checkoway, PhD, 4 Pierre Droz, PhD, ${ }^{5}$ Larry Fine, MD, DrPH, ${ }^{3}$ Lawrence Fischer, PhD, ${ }^{6}$ Robert Harris, PhD, ${ }^{7}$

Timo Kauppinen, PhD, 8 Rodolfo Saracci, MD 9

The International Workshop on Retrospective Exposure Assessment for Occupational Epidemiology Studies was held on 27-31 March 1990 at Leesburg, Virginia, in the United States. The presentations and discussions focused on the selection of an approach for assessing exposures, methods of exposure assessment used in industry-based and community-based studies, the evaluation of exposure estimates through measurements of validity and precision, and areas of future research.

\section{Background}

Retrospective exposure assessment in occupational epidemiology is a developing discipline that is important for the interpretation of causality in epidemiologic studies. Retrospective assessments are crucial in occupational studies of chronic diseases because these diseases are often characterized by long induction and latency periods. For such outcomes the most relevant exposures may have occurred prior to the 1970s, when environmental conditions were different than they are today, and relatively few occupational exposure measurements are available. Thus, for populations of workers exposed more than two or three decades ago, historical exposure measurements available today are rarely sufficient to permit a calculation of exposure levels for all members of the study population. Judgments must be made, therefore, of the historical exposures of workers in jobs without measurements and these judgments are generally based on qualitative and quantitative information indirectly related to exposure

\footnotetext{
1 Environmental Epidemiology Branch, National Cancer Institute, Rockville, Maryland, United States.

2 Program chair.

3 National Institute for Occupational Safety and Health, Cincinnati, Ohio, United States.

${ }^{4}$ University of Washington, Seattle, Washington, United States.

5 Institute of Occupational Medicine, Lausanne, Switzerland.

6 Michigan State University, East Lansing, Michigan, United States.

7 University of North Carolina, Chapel Hill, North Carolina, United States.

8 Institute of Occupational Health, Helsinki, Finland.

" International Agency for Research on Cancer, Lyon, France.
}

levels. Exposure estimates made from such information may have differing degrees of uncertainty and may be the subject of controversy because validated assessment methods have not been established. The Leesburg workshop was held to discuss the role of retrospective exposure assessments in epidemiologic studies, the current practice with respect to such assessments, and the prospects for improving them in future studies. This report summarizes the organizing committee's conclusions on the salient discussions, areas of consensus, and recommendations.

In many of the landmark studies of occupational hazards, quantitative assessments of exposure were rarely developed. Workers were typically classified by surrogate measures of exposure, such as employment by job or industry and duration of employment. In these studies, large risks of disease were associated with many of the hazardous agents studied. Quantitative exposure assessment was less critical, therefore, because misclassification of exposure was unlikely to hide an association completelv.

Many investigators have expressed concern, however, that these traditional methods of exposure assessment may be inadequate to reveal the more subtle effects of the hazardous agents of interest today, particularly those of chemicals. When true risks are small, observed relative risks can be easily overwhelmed by a small amount of exposure misclassification, and exposure classifications relying on ever/never exposed and duration of employment may not be sufficiently accurate to detect such risks (1). It is the committee's belief that these surrogate measures are inappropriate for current studies because of the danger of false negative results. Failure to develop quantitative estimates may result in a substantial underestimation of risks away from the heavily exposed because exposure misclassification dilutes exposure-response gradients, for steep gradients as well as modest ones. The committee suggests that these crude types of assessments may be useful in surveillance systems or in hypothesis generating studies. They should rarely be used, however, in hypothesis-testing studies, and then only after careful consideration of their limited usefulness in the evaluation of adverse exposures which cause small increases in relative risks. To reduce the misclassification of ex- 
posure by these surrogate measures, the committee recommends that quantitative exposure assessment be attempted in occupational epidemiologic studies wherever possible. Development of procedures for a detailed exposure assessment should begin with a determination, in the feasibility stages of a study, as to whether a quantitative evaluation of exposures is possible and whether the population is exposed to levels likely to result in the outcome of interest $(2,3)$.

\section{Toxicology and biological monitoring}

Toxicologic information can guide researchers with regard to the type of exposure measures or types of analyses that are most relevant to the disease(s) of interest. For example, cumulative and peak measurements of exposure may be valid and appropriate measures of disease risk, depending on the mechanistic basis of the toxicity of the disease in question $(4,5)$. Toxicologic factors which should be considered when decisions are made on the appropriate exposure measure to be assessed include the rate of entrance and elimination of the chemical to and from the body, the extent and rate of repair processes, and the time course of the development of the toxicity or disease after the initiating event. Consideration of these factors should be an integral part of the discussions concerning the collection of industrial hygiene samples and the development of estimates of exposure in an epidemiologic study. By accounting for these mechanisms, investigators are likely to enhance the ability to find an association if one truly exists.

Toxicologic information is, however, often lacking, contradictory, or ambiguous. In such situations, investigators may want to evaluate disease risks in relation to several different exposure measures to reduce the likelihood of choosing the incorrect toxicologic model and thereby miss an association $(1,6)$. This approach is easiest to apply when quantitative measurements or estimates of exposure, such as air concentrations in parts per million, have been developed.

In situations in which the toxicology of an agent is poorly understood, biological monitoring can provide valuable information (4), and sampling a small group of the study population can give insight into the relationship between the specific exposure situation of the study population and the dose received by this population. It may be useful for the investigators to review reports which compare environmental and biological monitoring results to determine whether the agent accumulates in the body or whether elimination or repair is rapid. From this information, the investigators can select the most appropriate biological marker. If cumulative exposure is the measure of interest under study, the biological monitoring method should be one which is an indicator of cumulative exposure $(5,7)$ (ie, one that measures an exposure marker with a long halftime). Alternatively, a biological method measuring a short half-time would provide information on current exposures and may be more relevant to evaluations of acute diseases. Even if the biological monitoring performed by the study investigators is not extensive enough to be relied upon exclusively to rank study subjects by exposure level, it can provide an indication of whether the exposure assessment for the jobs monitored appears reasonable. Biological monitoring may also be useful to assess absorption from exposures that occur by routes other than inhalation. This capability may be critical because current techniques for measuring and assessing exposures received by these other routes are less well developed than those for inhalation. For some agents, such as pesticides, these ancillary routes may actually be far more important than airborne exposures. A model which could be used for exposure assessment from dermal absorption is available (7), but there is need for more research on the most important predictors of dose from these other routes, such as duration of exposure, and the influence that area and the nature of contact have on the delivered dose.

Biological monitoring is the ideal technique for measuring the dose received because it gets closer to the target organ than exposure monitoring. Nevertheless, in the absence of unambiguous pharmacokinetic information, the committee believes that results from biological monitoring should not be accepted uncritically for several reasons. First, the mechanisms of distribution, metabolism, storage, and elimination of many agents in the body are very complex, and the biological marker selected may not be reflective of the exposure to the cells or tissues exhibiting the chemicalinduced damage. Second, different biological techniques for the same substance may classify subjects differently (7). Therefore, unless the toxic mechanism of the disease is well understood, biological monitoring should usually be accompanied by an assessment of exposure. Third, currently, there are few biological methods which reflect exposures from the distant past, such as those of concern in studies of cancer and other chronic diseases. In addition, biological monitoring is expensive and usually is and has been even less extensive than air monitoring. This scarceness makes extrapolation to persons not monitored difficult, particularly for the early years. Nevertheless, the committee believes investigators may wish to supplement industrial hygiene monitoring with some biological monitoring, particularly when there are multiple routes of exposure. The committee encourages the development of well-validated biological monitoring techniques and recommends including data from biological monitoring in exposure assessments whenever possible.

\section{Environmental monitoring and estimation of exposures}

In the absence of a clear interpretable biological marker to assess delivered dose to the organ of interest and 
a feasible technique to measure it, industrial hygiene monitoring of the environment will continue to be used as a surrogate for monitoring dose. Although use of industrial hygiene monitoring is more frequently seen in epidemiologic studies than biological monitoring is, it nevertheless has several limitations. Industrial hygiene monitoring data are typically unavailable for most agents prior to 1970 , and monitoring data are sparse for many substances even today (8). Furthermore, despite the anticipated increased emphasis on occupational health in the future, it seems likely that there will always be jobs and agents which will not have been monitored. Another limitation is that monitoring has generally been performed historically for purposes of compliance, rather than for the assessment of representative exposures of individuals. It is not known how sampling results from this type of sampling strategy reflect typical exposures (8), but, if they do not, relying on them to estimate exposures may result in incorrect estimates of disease risk. In addition, there is a lack of information on the nature of exposures, both to individual workers and to working populations (9). For example, little is known about the exposure distributions of an individual worker over time or the distributions of exposures among individual workers in a supposedly "homogeneous" exposure category, nor is it known how these two sources of variability affect epidemiologic results (8). By way of illustration, the workshop participants' analyses of the variability of exposure produced contradictory results. Investigators of a study in the cattle feed industry found that the variability of exposures between jobs was greater than the variability of exposure within jobs (10). In a similar comparison in several other industries, however, other researchers reported that the variation was often greater within than between jobs (5).

It seems likely that, in the near future, because of the insufficient number of biological and environmental monitoring data, quantitative exposure assessments for most agents will continue to require a substantial amount of judgment. Research is needed in the field of industrial hygiene (i) to characterize full-shift exposure distributions by determining the factors which generate log-normally distributed exposures and the circumstances when full-shift exposures are not lognormally distributed, (ii) to characterize the exposure distributions for individual job tasks, and (iii) to characterize the factors which influence exposure levels (11) and their interaction. Currently, the influences of the source's output strength, distance to the source, air motion rates, and other workplace characteristics are not well understood and quantified; hence, this is, the committee believes, an area where methodological studies may make significant contributions to retrospective exposure assessment procedures. The committee envisions in the future that algorithms will be available which use such factors in the process of assigning exposures, and therefore recommends that investigators evaluate monitoring data to quantify the influence of these factors on exposure levels. It also recommends that employers routinely document the important exposures experienced by workers and that monitoring surveys include representative sampling of all jobs, work areas, and tasks. Such information can then be used to characterize exposures and to develop exposure profiles which will reflect the typical exposures of workers and increase the accuracy of exposure estimates developed in future studies.

\section{Evaluation of exposure assessments}

In addition to the uncertainty surrounding measurements of exposures, another major weakness of current methodologies for exposure assessment is the issue of validity and reliability regarding subjects' recall of occupations and workplace exposure. Considerable work has been done on how well subjects recall information on diet and smoking, but there have been relatively few studies evaluating the effect of recall on the reporting of work histories $(12-14)$ or exposures from jobs. Even less has been done to assess how well employees can recall specific details of the environment in which exposures occurred many years ago. The ability to recall jobs held, exposures experienced, and environmental conditions may call upon different types of memory and require different interviewing techniques to elicit the information successfully. Little is known as to how occupational health investigators can create and ask questions in a way which best stimulates the interviewee's memory. Work is needed to determine the best approach for enabling workers to describe their work conditions in such a way as to allow an industrial hygienist to obtain an accurate picture of past exposures. Research conducted in the social sciences field may perhaps provide some insight into this area.

In cohort studies, the approach to obtaining detailed exposure information has become more standardized, and exposure estimates are becoming more quantitative in nature. In community-based case-referent studies, however, the techniques for collecting information and the assessment of exposures is much cruder. In many studies, exposure assessment is typically performed by a researcher, not always even an industrial hygienist, who assigns specific exposures primarily on the basis of job and industry titles, as reported by the subject. Another approach is to assess exposures for the subjects' jobs, on the basis of a previously developed (a priori) job-exposure matrix or on an ad hoc basis according to information provided by the subject (a posteriori). Although use of an a priori job-exposure matrix may be an improvement over analyses based simply on occupational categories, it is still subject to considerable misclassification (13). Extensive probing to obtain detailed descriptions of the work conditions of all subjects, however, is expensive and time consuming $(15,16)$. Rather than an extensive investigation of each subject's exposure 
history, it might be more practical to combine an a priori job matrix with detailed probing of selected exposed jobs. Alternatively, it has been indicated that it may be only the exposures assigned a positive assessment which need to be validated (14). This is an encouraging finding. With such an approach, exposure assessments could be improved by contacting only the subjects or the employers of the subjects positively exposed, and the process would be less expensive than performing detailed evaluations for all the subjects. Other creative ways of addressing this problem are needed.

In many epidemiologic reports, the description of the methods used by investigators to assess exposures have not been described in adequate detail. The committee believes such descriptions are insufficient in situations in which quantitative assessments have been developed. Investigators need to describe fully which exposure factors were evaluated, the weighting of the factors in the assessment of jobs, and their use to derive a final assessment. When exposure categories are used, the categories should be clearly defined, for example, as a minimum duration and/or level of exposure, particularly if the exposure can also occur outside the workplace (6). Examples of how the assessments were performed (ie, the model used) and examples of the results of the assessment across different exposure levels should be provided. This effort may require a separate exposure assessment report, but it is needed before the epidemiologic results can be evaluated fully and a better understanding of conflicting results from different studies can be provided.

Similarly the committee encourages investigators to develop as quantitative estimates of exposures as possible, taking maximum advantage of the available exposure information. If industrial hygiene and/or biological monitoring data are available, either for the study subjects or for a comparable population, the appropriateness of a detailed exposure assessment should be evaluated. The committee envisions that, in the future, criteria may be developed which will guide investigators as to when quantitative estimation of exposures is justifiable, how exposure estimates can be developed, and which assessment methods are most reasonable. An initial attempt has been made to develop such guidance through the identification of the attributes and characteristics necessary for a valid exposure assessment (2).

The committee also believes that, wherever possible, assessment methods should be evaluated for possible sources of misclassification (17). Evaluation of the reliability and validity of the exposure information $(13,14)$ and of the assessments $(18)$ can be used to construct the possible range of the true risk of the disease. Reliability of the assessment methods (ie, how different assessors compare among themselves) is generally possible and feasible, and it requires as little as a second assessor evaluating a sample of the jobs. Validity of the assessment procedures (ie, how the assessors compare with the "truth") is more difficult because the truth is not known. Several methods of evaluating the reliability and validity of exposure estimates have been made (18).

The committee believes that the development and improvement of techniques for the quantitative assessment of historical exposure levels will be increasingly important in occupational epidemiologic research. It is encouraging to see that the European Economic Community has identified areas of research in retrospective exposure assessment that it intends to investigate formally (19). The committee recommends to other occupational health scientists that they also conduct methodological studies on these issues wherever possible, however small. The sharing of such information will allow other scientists to critique and improve the methods in use, and it is only by doing so that the field will develop into an accepted scientific discipline. Substantial progress has been made in the 1980 s, and the committee is hopeful for the 1990 s.

\section{References}

1. Stewart $\mathbf{P}$, Herrick R. Issues in performing retrospective exposure assessment. Appl Occup Environ Hyg 1991;6:421-7.

2. Esmen N. Analysis of strategies for reconstructing exposures. Appl Occup Environ Hyg 1991;6:488-94.

3. Park R, Silverstein M, Mirer F. Characteristics of worker populations: exposure considerations in the selection of study populations and their analysis. Appl Occup Environ Hyg 1991;6:436-40.

4. Axelson $O$, Soderkvist P. Characteristics of disease and some exposure considerations. Appl Occup Environ Hyg 1991;6:428-35.

5. Rappaport S. Selection of the measures of exposure for epidemiology studies. Appl Occup Environ Hyg 1991; 6:448-51.

6. Kauppinen, T. Development of a classification strategy of exposure for industry-based studies. Appl Occup Environ Hyg 1991;6:482-7.

7. Droz P, Berode M, Wu M. Evaluation of concomitant biological and air monitoring results. Appl Occup Environ Hyg 1991;6:465-74.

8. Harris R. Measures to facilitate future epidemiologic studies. Appl Occup Environ Hyg 1991;6:555-8.

9. Smith T, Hammond S, Hallock M, Woskie S. Retrospective exposure assessment for epidemiology: characteristics of exposure. Appl Occup Environ Hyg 1991;6: $441-7$.

10. Heederik D, Boleij J, Kromhout H, Smid T. Use and analysis of exposure monitoring data in occupational epidemiology. Appl Occup Environ Hyg 1991;6:45864.

11. Schneider T, Olsen I, Jorgensen O, Lauersen B. Evaluation of exposure information. Appl Occup Environ Hyg $1991 ; 6: 475-81$.

12. Bond G, Bodner K, Olsen G, Burchfiel C, Cook R. Validation of work histories for the purpose of epidemiological studies. Appl Occup Environ Hyg 1991;6:521-7.

13. Miligi L, Masala G. Methods of exposure assessment for community-based studies: aspects inherent to the validation of questionnaires. Appl Occup Environ Hyg 1991;6:502-7.

14. Stewart W, Correa-Villasenor A. False positive exposure errors and low exposure prevalence in community-based case-control studies. Appl Occup Environ Hyg 1991;6: 534-40.

15. Gerin M, Siemiatycki J. The occupational questionnaire 
in retrospective epidemiologic studies: recent approaches in community-based studies: Appl Occup Environ Hyg 1991;6:495-501.

16. Dewar R, Siemiatycki J, Gerin M. Loss of statistical power associated with the use of a job exposure matrix in occupational case-control studies. Appl Occup Environ Hyg 1991;6:508-15.

17. Checkoway H, Savitz D, Heyer N. Assessing the effects of non-differential misclassification of exposures in oc- cupational studies. Appl Occup Environ Hyg 1991;6: $526-33$.

18. Hornung R. Statistical evaluation of exposure assessment strategies. Appl Occup Environ Hyg 1991;6:51620.

19. Hemon D, Berrino F, Brochard P, et al. Retrospective evaluation of occupational exposures in cancer epidemiology: a European concerted action of research. Appl Occup Environ Hyg 1991;6:541-6. 